\title{
Endometrial Scratching Effect on Pregnancy Rate in Intrauterine Insemination Cycles: A Randomized Controlled Trial
}

\author{
Mina Naghi Jafarabadi ${ }^{1}$, Maryam Bagheri $^{2}$, Zahra Ebrahimi $^{3^{*}}{ }^{\circledR}$, Mamak Shariat $^{4}$, Fedyeh Haghollahi $^{5}$
}

\begin{abstract}
Objectives: This study was aimed to examine the endometrial scratch injury effect to improve pregnancy rate in women undergoing intrauterine insemination (IUI).

Materials and Methods: This randomized controlled clinical trial was conducted in a University Clinic from November 2017 to January 2019. A total of 120 candidates entered the study, who were assigned to intervention $(n=60)$ and control $(n=60)$ groups. All subjects underwent controlled ovarian stimulation using letrozole $2.5 \mathrm{mg} / \mathrm{BD}$ on a day between 3 and 7 of the cycle. Then, the sonographic monitoring was started from day 8 of the cycles, followed by injecting human chorionic gonadotropins (HCG) 100001U intramuscularly when 1-2 follicles reached $18 \mathrm{~mm}$ in diameter. Approximately 36-38 hours afterward, IUI was done and a B-HCG test was performed after 15 days as well. In addition, luteal phase support was performed with the vaginal progesterone $400 \mathrm{mg}$ twice daily for 15 days. In the intervention group, on day 3 of the cycle endometrial cavity on the posterior wall was scratched with the vaginal cannula No. 4 by a single infertility specialist in addition to the routine procedure. The chemical and clinical pregnancy rates were the main outcome measurements.

Results: The overall clinical pregnancy rates were $11 / 59$ (18.6\%) and 10/59 (16.9\%) in intervention and control groups, respectively $(P=0.810)$. Further, the abortion rates were $1 / 59(1.7 \%)$ and $3 / 59(5.1 \%)$ in intervention and control groups, respectively $(P=0.303)$. Eventually, no cases of ectopic pregnancy were observed in either group.

Conclusions: Based on the findings of this study, endometrial scratch by pipelle cannot improve pregnancy rate in the early proliferative phase.

Keywords: Infertility, Pregnancy, Endometrial receptivity, Endometrial scratching
\end{abstract}

\section{Introduction}

Intrauterine insemination (IUI) is considered as one of the most common types of artificial insemination, through which controlled ovarian stimulation may or may not be used. This method is used to treat various types of infertility such as male factor, cervical factor, ovulation disorder, mild endometriosis (which does not involve fallopian tubes), and infertility with unknown causes (1). In addition, it is chosen as the first-line treatment for infertility due to the low cost and less invasive nature. According to various studies, the incidence of pregnancy rate is $7 \%$ to $22 \%$ in each period (2) although very low (4\%) and high pregnancy $(40 \%)$ rates in IUI are not reported as well $(3,4)$.

Sperm health indicators including the number, morbidity and morphology, and improvement of implantation are considered among success factors in the IUI $(5,6)$. The improvement of implantation is one of the most commonly used treatments for infertility with a complex and multidimensional process including an increase in growth factor and cytokines that regulate inter-embryo and endometrial interactions (7).

The successful implantation of a fetus requires two factors such as a healthy embryo and a healthy endometrium. Any kind of embryonic anomalies, endometrium or immune system defects leads to an implantation insufficiency (8).

Further, endometrial mechanical manipulation increases the endometrial receptivity by altering the gene expression of laminin alpha 4 genes, alpha 6 integrin, metalloprotein matrix, and glycollin (9) and an increase in implantation may be due to endometrial regeneration as well. Endometrial scratching is an affordable and tolerable method in patients who are in vitro fertilization (IVF) candidates, which increases the likelihood of pregnancy and birth rate. Barash first reviewed this issue in his study and concluded that women who underwent IVF and received a biopsy experienced pregnancy rates twice as high as the control group (10).

However, the data on the effect of endometrial scratching in IUI is insufficient. Further, studies focusing on the

Received 1 April 2019, Accepted 3 August 2019, Available online 25 August 2019

${ }^{1}$ Vali-Asr Reproductive Health Research Center, Tehran University of Medical Sciences, Tehran, Iran. ${ }^{2}$ Department of Reproductive Health, School of Nursing and Midwifery, Tehran University of Medical Sciences, Tehran, Iran. ${ }^{3}$ Ziaeian Hospital, Tehran University of Medical Sciences. Tehran, Iran. ${ }^{4}$ Family Health Institute, Maternal-Fetal and Neonatal Research Center, Tehran University of Medical Sciences, Tehran, Iran. ${ }^{5}$ Reproductive Health Research Center, Tehran University of Medical Sciences Tehran, Iran.

*Corresponding Author: Zahra Ebrahimi, Tel: +989123838645, Email: drebrahimi1234@yahoo.com 
endometrium in IUI/IVF cycles revealed that there is an inconsistency in the days of injury to the endometrium, scratching methods, and the number of the required scratches. Thus, the current study aimed to assess the rate of pregnancy using endometrial scratch on the third day of the cycle in infertile couples opting for the IUI.

\section{Materials and Methods}

The present randomized clinical trial study was conducted at Vali-Asr Infertility Clinic in Imam Khomeini Hospital, Tehran between November 2017 and January 2019. After giving informed consent, 120 women candidates for IUI entered the study and were divided into intervention and control groups.

The inclusion criteria included being as women with primary or secondary infertility with unknown causes, being within the age range of 21-35 years, having a body mass index (BMI) of 18-30, having a normal hormonal profile $(\mathrm{FSH}<10)$ and thyroid test, having no adnexal mass in ultrasound examination, and being in the menstrual cycle of 25-31 days. Cases of abnormal prolactin, myoma, and systemic disease were excluded from this study. Both groups were matched in terms of age, infertility duration, and history of IUI or IVF.

All the study subjects underwent controlled ovarian stimulation using letrozole. In both groups, ovulation was induced by letrozole (Tehran Chimi Company, Iran) 2.5 $\mathrm{mg} / \mathrm{BD}$ on days 3 to 7 of the cycle. Then, follicle monitoring was started from day 8 of the cycle. In addition, HCG $100001 \mathrm{U}$ (Choriomon, IBSA) was injected intramuscularly when 1-2 follicles reached $18 \mathrm{~mm}$ or over in diameter, followed by performing IUI 36-38 hours after injection, as well as a pregnancy test 15 days later. Luteal phase support was performed with the vaginal progesterone (Cyclogest, Actover Company, Iran) $400 \mathrm{mg}$ twice daily for 15 days. In the intervention group, the endometrial cavity on the posterior wall was scratched with the vaginal cannula No. 4 (Vitaimed Instrument Company, LTD, Iran) by a single infertility specialist on day 3 of the cycle in addition to the routine procedure. The main outcome measurements were the rate of chemical and clinical pregnancy and the secondary outcome measurements included the abortion and ectopic pregnancy rate. Patients with a positive pregnancy test were followed up to 20 weeks of pregnancy. However, those with negative pregnancy tests were allowed to try spontaneous conception for one cycle. Chemical pregnancy rate and clinical pregnancy (viable intrauterine pregnancy by ultrasound) were the primary outcomes. Moreover, the rates of the loss of pregnancy and ectopic pregnancy were considered as secondary outcomes.

\section{Statistical Analysis}

The SPSS 19 package program (SPSS Inc, an IBM Company) was used to do all the statistical analyses. Furthermore, the Mann-Whitney test was utilized for data with no normal distribution and the chi-squared test was used for qualitative variables. The $P<0.05$ was considered statistically significant.

\section{Results}

A total of 120 patients suffering from primary or secondary infertility were studied in 2 intervention $(n=60)$ and control $(n=60)$ groups. Of these, the cycles of 2 subjects were cancelled, including one in the intervention and the other in the control groups due to inducing multiple mature follicles and no response to induction (Figure 1).

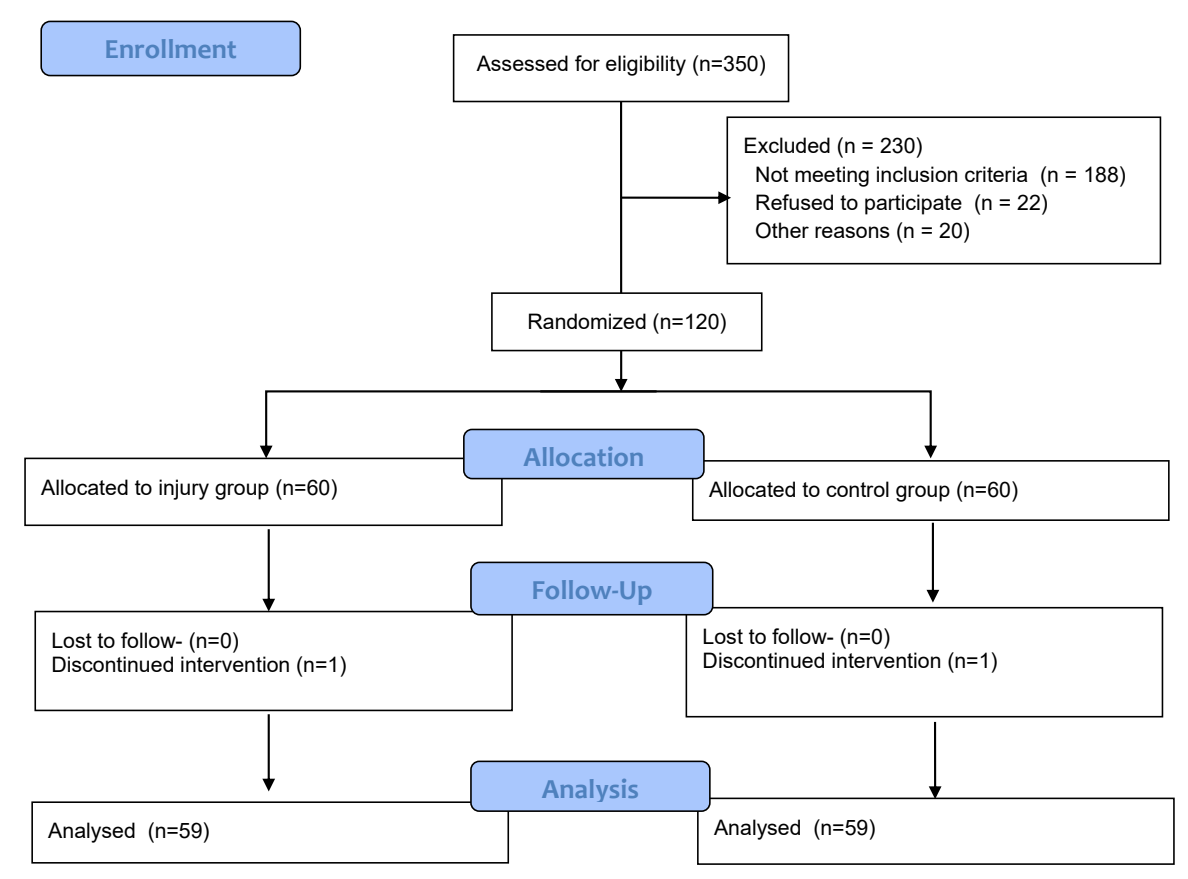

Figure 1. Consort Flow Diagram. 
The participants in both groups were comparable with respect to age, BMI, and other baseline parameters (Table 1). The mean endometrial thickness on the third day of the cycle was the same between the intervention and control groups. However, the mean of the endometrial thickness (millimeter) measured on the day of the trigger was higher $(P=0.036)$ in the intervention group (Table 2$)$.

The chemical pregnancy rates in intervention and control groups were 12/59 (20.3\%) and 10/59 (16.9\%), respectively, and thus the difference was not statistically significant $(P=0.636)$, the details of which are provided in Table 3.

The overall clinical pregnancy rates were $11 / 59$ (18.6\%) and $10 / 59$ (16.9\%) in intervention and control groups, respectively, and the difference was not statistically significant $(P=0.810)$. Furthermore, the abortion rates were $1 / 59(1.7 \%)$ and $3 / 59(5.1 \%)$ in the intervention and control groups, respectively $(P=0.303)$. Finally, no cases of ectopic pregnancy were found in either group.

\section{Discussion}

The present study was aimed to investigate the role of endometrial scratching in infertile couples undergoing ovulation induction in the IUI cycles. The results of the study showed no significant difference in chemical and clinical pregnancy, abortion, and ectopic pregnancy rates in patients undergoing endometrial scratching in the early proliferative phase during the stimulation cycle. However, other studies demonstrated a significantly higher clinical pregnancy rate in patients which endometrial scratching in the mid proliferative phase of stimulation in the IUI cycle $(7,11,12)$.

Similarly, Soliman and Harira found a significantly higher biochemical, clinical, and ongoing pregnancy rate in the scratching group in the mid proliferative phase of the IUI cycle (13). The results of these studies (7,11-13) are not consistent with those of the present study.

In the study of Zarei et al, endometrial biopsy was performed on a day between 6 and 8 of the cycle before the IUI. The results indicated that the local damage to endometrium in the cycle before the IUI is not associated with an increase in pregnancy and abortion rates or endometrial thickness (14). In the clinical trial by El-Khayat et al, the endometrial injury was done using hysteroscopy and tooth forceps. The results demonstrated no significant difference in clinical pregnancy rates in endometrial scratching compared with performing hysteroscopy in female candidates of the IUI (15).

In addition to interventional studies (7,11-15), a review article study was conducted and its results suggested no probability of an increase in pregnancy rate in the IUI cycles and it was recommended to conduct larger sample size clinical studies (16). Another systematic review and meta-analysis suggested that endometrial scratch injury performed once, preferably during the follicular phase of the same cycle of the IUI with flexible aspiration catheters,

Table 1. Baseline Characteristics of the 2 Groups

\begin{tabular}{|c|c|c|c|}
\hline Characteristics & Injury Group ( $n=60)$ & Control Group $(n=60)$ & $P$ Value $^{\mathrm{a}}$ \\
\hline Age $(y)$, (Mean \pm SD) & $28.66 \pm 4.33$ & $29.33 \pm 3.65$ & 0.526 \\
\hline Education $^{\mathrm{b}}$, No. (\%) & & & 0.308 \\
\hline Lower diploma & $41(68.3 \%)$ & $33(55 \%)$ & \\
\hline Diploma & $14(23.3 \%)$ & $31(35 \%)$ & \\
\hline Higher diploma & $5(8.3 \%)$ & $6(10 \%)$ & \\
\hline BMI $\left(\mathrm{kg} / \mathrm{m}^{2}\right)$, (Mean $\left.\pm \mathrm{SD}\right)$ & $25.65 \pm 2.74$ & $25.71 \pm 2.84$ & 0.875 \\
\hline Duration of infertility $(y)$, (Mean $\pm S D$ ) & $3.25 \pm 1.95$ & $3.50 \pm 2.03$ & 0.53 \\
\hline Type of infertility ${ }^{b}$ No. (\%) & & & 0.673 \\
\hline Primary & $46(76.7 \%)$ & $44(73.3 \%)$ & \\
\hline Secondary & $14(23.3 \%)$ & $16(26.7 \%)$ & \\
\hline Number of previous IUI (n) & $0.23 \pm 0.46$ & $0.31 \pm 0.53$ & 0.385 \\
\hline Number of previous IVF ( $\mathrm{n}$ ) & 0 & 0 & 0.156 \\
\hline $\mathrm{AMH}(\mathrm{ng} / \mathrm{mL})$ & $2.44 \pm 0.54$ & $2.50 \pm 0.63$ & 0.955 \\
\hline
\end{tabular}

SD: Standard deviation; BMI: Body mass index; IUI: Intrauterine insemination; IVF: In vitro fertilization; AMH: Anti-Müllerian hormone.

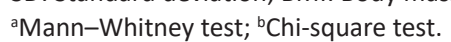

Table 2. Clinical Characteristics After Intervention in the 2 Groups

\begin{tabular}{|c|c|c|c|}
\hline & Intervention Group ( $n=59$ ) & Control Group $(n=59)$ & $P$ Value $^{\mathrm{a}}$ \\
\hline Endometrial thickness $(\mathrm{mm})$ in day of cycle (Mean \pm SD) & $3.41 \pm 0.48$ & $3.30 \pm 0.44$ & 0.236 \\
\hline Endometrial thickness $(\mathrm{mm})$ in the day of HCG injection (Mean \pm SD) & $8.65 \pm 0.76$ & $8.25 \pm 1.37$ & 0.036 \\
\hline Number of follicles $\geq 18 \mathrm{~mm}(\mathrm{n}$ ) on day of HCG injection (Mean \pm SD) & $1.18 \pm 0.39$ & $0.70 \pm 0.76$ & 0.0001 \\
\hline
\end{tabular}

SD: Standard deviation; HCG, Human chorionic gonadotropins.

aMann-Whitney test. 
Table 3. Pregnancy Rate in the 2 Groups

\begin{tabular}{lccc}
\hline & $\begin{array}{c}\text { Intervention } \\
\text { Group }(\mathbf{n = 5 9 )}\end{array}$ & $\begin{array}{c}\text { Control } \\
\text { Group }(\mathbf{n = 5 9 )}\end{array}$ & P Value $^{\mathrm{a}}$ \\
\hline Chemical pregnancy rate & $12(20.3 \%)$ & $10(16.9 \%)$ & 0.636 \\
Clinical pregnancy rate & $11(18.6 \%)$ & $10(16.9 \%)$ & 0.810 \\
Abortion rate & $1(1.7 \%)$ & $3(5.1 \%)$ & 0.309 \\
\hline
\end{tabular}

Note. Data are presented as numbers (\%).

${ }^{a}$ Chi-square test.

may improve clinical pregnancy and ongoing pregnancy rates in IUI cycles (17).

Different findings and contradictory results of the above-mentioned studies are supposed to be related to the differences in the design, sample sizes, and the method of conducting the studies. In these studies, the time of creating the scratch was different, which was probably effective in the outcome. In addition, the probable reason may be the longer duration between scratching and the IUI, leading to the limited benefit of endometrial injury. In some other different studies $(7,11,12)$, scratching was done on different days of the previous luteal or proliferative phase of the menstrual cycle prior to the IUI cycle. Moreover, different factors influencing the IUI outcome, including women's age, BMI, need future confirmation by randomized trials (16).

However, further studies are required to clarify this inconsistency. Furthermore, the pipelle was employed in this study while biopsy or other methods of endometrial scratching were adopted in some previous studies. so far, the proof is still insufficient regarding the benefits of endometrial scratching in the IUI and assisted reproductive treatment cycles because of the limited sample size and the poor quality of the studies (10). Therefore, if the results of the future study show that localized endometrial injury in infertile couples choosing IUI increases the pregnancy rates, thus it can be carried out prior to other therapeutic interventions, reducing the cost of treatment and psychological stress in infertile couples during infertility treatment.

The low sample size is considered as the main limitation of the present study.

\section{Conclusions}

According to the findings of this study, the endometrial scratch cannot improve pregnancy rate using pipelle in the early proliferative phase. The final decision on whether to apply endometrial scratches requires more extensive clinical trials.

\section{Conflict of Interests}

The authors have no competing interests that might be perceived to influence the results and discussion reported in this paper.

\section{Ethical Issues \\ This prospective randomized trial was approved}

by The Ethics Committee of Tehran University of Medical Sciences (IR.TUMS.IKHC.REC.1397.533) and was registered at Iranian Registry of Clinical Trials (identifier: IRCT20180624040214N1; https://www.irct.ir/ trial/32114).

\section{Financial Support}

This paper as a fellowship thesis was funded by the Deputy of Research, Tehran University of Medical Sciences, Tehran, Iran.

\section{References}

1. Yavuz A, Demirci O, Sözen H, Uludoğan M. Predictive factors influencing pregnancy rates after intrauterine insemination. Iran J Reprod Med. 2013;11(3):227-234.

2. Ashrafi M, Rashidi M, Ghasemi A, et al. The role of infertility etiology in success rate of intrauterine insemination cycles: an evaluation of predictive factors for pregnancy rate. Int J Fertil Steril. 2013;7(2):100-107.

3. Intrauterine insemination. Hum Reprod Update. 2009;15(3):265-277. doi:10.1093/humupd/dmp003

4. Requena A, Herrero J, Landeras J, et al. Use of letrozole in assisted reproduction: a systematic review and metaanalysis. Hum Reprod Update. 2008;14(6):571-582. doi:10.1093/humupd/dmn033

5. Dekel N, Gnainsky Y, Granot I, Mor G. Inflammation and implantation. Am J Reprod Immunol. 2010;63(1):17-21. doi:10.1111/j.1600-0897.2009.00792.x

6. Yalti S, Gürbüz B, Sezer H, Celik S. Effects of semen characteristics on IUI combined with mild ovarian stimulation. Arch Androl. 2004;50(4):239-246. doi:10.1080/01485010490448435

7. Wadhwa L, Pritam A, Gupta T, Gupta S, Arora S, Chandoke R. Effect of endometrial biopsy on intrauterine insemination outcome in controlled ovarian stimulation cycle. J Hum Reprod Sci. 2015;8(3):151-158. doi:10.4103/09741208.165144

8. Mirkin S, Arslan M, Churikov D, et al. In search of candidate genes critically expressed in the human endometrium during the window of implantation. Hum Reprod. 2005;20(8):2104-2117. doi:10.1093/humrep/dei051

9. Zhou L, Li R, Wang R, Huang HX, Zhong K. Local injury to the endometrium in controlled ovarian hyperstimulation cycles improves implantation rates. Fertil Steril. 2008;89(5):1166-1176. doi:10.1016/j.fertnstert.2007.05.064

10. Barash A, Dekel N, Fieldust S, Segal I, Schechtman E, Granot I. Local injury to the endometrium doubles the incidence of successful pregnancies in patients undergoing in vitro fertilization. Fertil Steril. 2003;79(6):1317-1322. doi:10.1016/s0015-0282(03)00345-5

11. Abdelhamid AM. The success rate of pregnancy in IUI cycles following endometrial sampling. A randomized controlled study: endometrial sampling and pregnancy rates. Arch Gynecol Obstet. 2013;288(3):673-678. doi:10.1007/s00404013-2785-0

12. Goel T, Mahey R, Bhatla N, Kalaivani M, Pant S, Kriplani A. Pregnancy after endometrial scratching in infertile couples undergoing ovulation induction and intrauterine insemination cycles-a randomized controlled trial. J Assist Reprod Genet. 2017;34(8):1051-1058. doi:10.1007/s10815- 
017-0949-8

13. Soliman BS, Harira M. Local endometrial scratching under ultrasound-guidance after failed intrauterine insemination and cycle outcome: a randomized controlled trial. Middle East Fertil Soc J. 2017;22(1):60-66. doi:10.1016/j. mefs.2016.06.006

14. Zarei A, Alborzi S, Dadras N, Azadi G. The effects of endometrial injury on intrauterine insemination outcome: a randomized clinical trial. Iran J Reprod Med. 2014;12(9):649-652.

15. El-Khayat W, Elsadek M, Saber W. Comparing the effect of office hysteroscopy with endometrial scratch versus office hysteroscopy on intrauterine insemination outcome: a randomized controlled trial. Eur J Obstet Gynecol Reprod Biol. 2015;194:96-100. doi:10.1016/j.ejogrb.2015.08.025

16. Lensen SF, Manders M, Nastri CO, et al. Endometrial injury for pregnancy following sexual intercourse or intrauterine insemination. Cochrane Database Syst Rev. 2016(6):CD011424. doi:10.1002/14651858.CD011424. pub2

17. Vitagliano A, Noventa M, Saccone G, et al. Endometrial scratch injury before intrauterine insemination: is it time to re-evaluate its value? Evidence from a systematic review and meta-analysis of randomized controlled trials. Fertil Steril. 2018;109(1):84-96. doi:10.1016/j.fertnstert.2017.09.021

(c) 2020 The Author (s); This is an open-access article distributed under the terms of the Creative Commons Attribution License (http://creativecommons.org/licenses/by/4.0), which permits unrestricted use, distribution, and reproduction in any medium, provided the original work is properly cited. 